\title{
Application of Microwave Promoted Chemical Reactions on Pharmaceutical Synthesis
}

\author{
Lv Hongxia \\ Xi'an International University, Shaanxi, China, 710077
}

Keywords: Microwave technology, Chemical reaction, drug synthesis, Technology application

\begin{abstract}
In recent years, microwave technology has been widely used in chemical reactions. It brings new changes to chemical reactions, especially in drug synthesis. Microwave technology has many advantages in chemical reactions. How to use microwave technology to enhance the effect of chemical reactions is the focus of technical research.
\end{abstract}

\section{Introduction}

The wavelength of the microwave is usually 1-1000 mm, but the frequency is very high. It can reach 0.3-300 GHz. Starting from the beginning of the last century, a large number of scientists began to study microwave technology and obtained many breakthroughs. For example, it turns out that microwaves can pass through hollow metal tubes. Microwave and different substances interact to achieve the conversion of energy. Microwave heating can be efficient and clean. Microwave heating is different from the traditional heating. From the middle and late of the last century, scientists began to experimentally apply microwave technology to chemical synthesis and made many breakthrough achievements. The microwave conditions can be solved. Oxidation and hydrolysis and other reactions can improve the reaction rate. At present, due to the unique advantages of microwave technology, many chemical syntheses began to use microwave technology.

\section{Microwave technology heating principle}

Microwave heating is the dielectric effect through the microwave. Some of the solid or liquid can change the inherent electromagnetic energy and the heat generated during the transformation. The temperature is object. The principle of microwave heating is: under the action of microwaves, the internal molecules of the polar compounds will interact with each other, and the orientation of the molecules will tend to be consistent with the direction of the electric field. Electric field in the positive and negative conversion occurs, the polar compounds will be the role of the electric field. The polarity will change in order to maintain consistency with the role of the field. As the molecules move under the action of an electric field, the movement lags behind the change of the electric field. As a result, the molecules are distorted and the internal temperature of the compound rises and heating is achieved.

\section{Microwave technology to promote the chemical reaction mechanism}

Microwave technology was first applied to communications products as the technology matures. The chemical field began to use microwave technology to complete the relevant chemical reactions. Due to the application of microwave technology the chemical synthesis reaction enhances the reaction rate. Comparing with the traditional method, the reaction rate can be increased several hundred times to several thousand times. The current academic community has two different perspectives on the mechanism of action of microwaves.

A view that the microwave to take the heating method. You can achieve rapid heating to ensure uniformity of heating, eliminating the temperature gradient within the object. There is no hysteresis effect. Microwave technology used in chemical reactions only to achieve the heat supply. The 
traditional heating method is not too big difference. The frequency of microwaves used in chemical reactions is usually $2500 \mathrm{MHz}$. This heating is a non-ionizing radiation. The chemical bonds of the molecules resonate during the heating process. However, the resonance does not break the chemical bonds and the molecules do not change into high-level vibrations. Microwave acceleration of the chemical reaction is reflected in the choice of the polarity of organic matter. Microwave reaction and conventional heating compared to the reaction power will not change significantly.

The other point of view that the microwave on the chemical reaction will play a role under the action of microwave molecular motion will be more intense. The temperature will change. Microwaves have an effect on ions and polar molecules, and the relative motion between molecules will change. And this change is related to the frequency of microwaves, which will affect the temperature change. Therefore, microwaves have a complex mechanism for accelerating chemical reactions. Microwave will increase the activation energy of the reaction. The reaction will speed up, thus speeding up the chemical reaction process.

\section{Microwave reactor application}

Domestic microwave ovens have produced many results by retrofitting applications in chemical laboratories, but there are some drawbacks with this approach. Due to the limitations of domestic microwave ovens, the inability to provide accurate data for chemical reactions, some of which cannot be done in domestic microwave ovens, it can be achieved in dedicated microwave ovens. Home microwave oven defects mainly reflected in: due to the low microwave power, some of the chemical reactions demanding field strength cannot be achieved. The use of domestic microwave oven can not accurately obtain the actual temperature of the reaction occurs. The reaction state cannot be observed in real time. The heating zone of household microwave oven concentrated in the lower part of the furnace. And the synthesis of drugs need to be completed under stirring and the reaction should be kept evenly heated. So household Microwave ovens are difficult to implement and may cause side effects. Home microwave ovens take intermittent heating and heating power is affected by voltage.

\section{The advantages of microwave technology}

Through the material surface heat transfer the traditional principle of heating is heat conduction or heat radiation effect on the object. Microwave with the intermolecular interaction within the electromagnetic field can heat directly on the object. In the heat transfer mode, the traditional heating there will be a thermal gradient, thermal energy will shift. But microwave heating realizes the conversion of electromagnetic energy and thermal energy instead of heat transfer. For material heating, the microwave heating has many advantages due to the heat transfer achieved by microwave heating. Such as microwaves can penetrate the entire material and energy savings, heat can be generated from different parts of the material. The rate at which energy is transferred and it is independent of the material surface. And bulky materials can also heat quickly and achieve uniform heating. Especially for some of the compounds with low thermal conductivity, they can effectively improve the heating rate. The traditional heating method, the heating rate and material properties are related to the temperature gradient. But the use of microwave heating energy can be transmitted evenly, the temperature of various parts of the material there is no gradient. Therefore, shortening the reaction time and improve the reaction effect.

\section{Microwave chemical synthesis of the classification}

\subsection{Microwave closed synthesis technology}

In the middle of the last century, some scientists applied microwave technology to the synthesis of drugs. The synthesis reaction uses a closed reactor, placing all of the reactants in a closed reactor, placing in a microwave source, subjecting to microwave irradiation and then treating the compound. Studies have shown that under confined conditions, due to the action of microwaves, the drug 
synthesis reaction occurs rapidly and the reaction speed is accelerated due to the instantaneous generation of high temperature and pressure. However, under the action of high pressure, the reactor will be deformed in case of serious explosion. After the improvement, a reactor can regulate the internal pressure of the reactor. It was designed and the temperature of the chemical reaction can be controlled by controlling the pressure of the chemical reaction process. Subsequent research has been made under closed conditions microwave batch reactor. It can achieve rapid heating and microwave power can be realized without control.

\subsection{Microwave atmospheric synthesis technology}

In order to microwave technology applied to the synthesis of drugs under atmospheric pressure. In the middle and late last century, researchers tried microwave synthesis under normal pressure and successfully completed the synthesis of aspirin intermediates using this technology. During the synthesis, the reactants are placed in a container and placed in a microwave. It controls the microwave radiation during the reaction so as to raise the temperature of the reactants in a slow state. In addition, the microwave reaction vessel and an external condensing device phase, you can synthesize a variety of drugs. Due to the stirring and dropping effect, the synthesis conditions of the organic substance can be satisfied. And the microwave synthesis medicine can be completed under the normal pressure condition. Due to the simple operation, the microwave synthesis medicine has been widely applied.

\subsection{Microwave continuous synthesis reaction technology}

The maturity of the two synthetic technologies for the continuous synthesis is the reaction technology to create the conditions. Continuous synthesis reaction technology is the reaction liquid through the role of external power flow at a certain flow rate into the microwave reactor for continuous reaction. The earliest continuous microwave technology enabled the synthesis of esters and alcohols. However, this kind of reaction has some defects, such as the difficulty of measuring the temperature of the reaction process. The use of this device cannot guarantee the effect of the reaction. In particular, the synthesis reaction with strict control of the reaction temperature does not guarantee the quality. For example, the reaction containing solid content cannot be achieved. The viscosity of the liquid or under low temperature conditions of the reaction is difficult to achieve. In addition, taking into account the reaction of raw materials on the microwave energy incompatibility problems, such as the presence of metal or reactants are non-polar organic, microwave continuous reaction cannot be achieved.

\subsection{Microwave dry synthesis reaction technology}

Dry synthesis of organic matter under microwave conditions requires an inorganic support, usually porous materials such as alumina or diatomaceous earth. The reaction product is obtained by solvent extraction after reaction in a microwave oven. Since inorganic carriers do not absorb microwaves of specific frequencies, the organic substances attached to the surface of the carriers. They can fully acquire the microwave energy and ensure the complete reaction. The reactant molecules are fully activated by the microwave, so the reaction rate can be increased. The earliest microwave dry synthesis reaction by external gas source is achieved. During the reaction, blowing air can play a stirring effect to ensure that the reactants are uniformly mixed. After the reaction is completed, the gas source can be connected to the vacuum pump. Excess liquid generated during the reaction can be sucked off. The dry reaction needs to be carried out on the support, so the number of reactants will be limited. In addition, the choice of solid support has some limitations, affecting the scope of application of dry microwave synthesis.

\section{Microwave technology in the synthesis of drugs}

\subsection{In the case of organic drugs into the application}

Due to the particularity of the heat transfer method, microwave technology can be widely applied to the chemical synthesis of organic drugs and it can effectively shorten the reaction time. In 
addition, no additional solvent is required during the reaction, which is beneficial to reduce the production of reactants cost. At present, microwave technology plays an important role in the synthesis reactions as well as the chemical synthesis of heterocyclic compounds. Compared with the traditional method, microwave method has the advantages of simple operation, short reaction time and high production efficiency. In addition, you can also ensure the quality of the reaction and the purity of the reactants can be guaranteed.

\subsection{In the application of inorganic drug synthesis}

Traditional inorganic synthesis heating conduction method, but this has some drawbacks, such as low efficiency. Due to the heating by convection or heat conduction between materials to be completed, the reaction temperature is difficult to ensure uniformity. The use of microwave technology can effectively avoid this defect. The reactor can be uniform and uniform temperature. Therefore, microwave technology can enhance the effect of inorganic synthesis. The use of microwave technology can be uniform particle size, decentralized effect, high purity crystalline products.

\subsection{Analysis of drug synthesis}

Microwave technology in the analysis of drug synthesis can show many advantages. For example, the microwave sample-dissolving technology has the advantages of high efficiency, rapidness, uniform temperature and easy temperature control. It is particularly suitable for sample processing. Traditional sample analysis requires pretreatment time. Therefore, there is a time difference between drug analysis and sample preparation. The use of microwave technology can improve the accuracy and precision of drug analysis, which can effectively reduce the time required for determination. Therefore, measuring efficiency can be improved. You can achieve the energy saving of the analysis process.

\section{Microwave technology application prospects}

Currently, microwave technology has been used in many areas of chemical research. In drug synthesis, microwaves can promote chemical reactions. Due to the increased heating rate, the rate of chemical reaction can be increased. Microwave technology is used to achieve a non-contact heat source and reactants. It can accurately control the reaction process. The reaction of various parameters of the effective control can improve product quality. Microwave technology can also solve the safety problems in drug synthesis, which can reduce the incidence of side effects. Therefore, the application of the technology can realize the automation of drug synthesis. It can save time and improve efficiency and reduce environmental pollution. It has broad market application prospect. Microwave technology has promoted the application of chemical reactions in drug synthesis.

\section{Conclusions}

Microwave chemistry is a new discipline that crosses two disciplines. It has many advantages that can be applied to drug synthesis, which can bring a broader application prospect. The application of microwave technology improves the reaction speed. It has the advantages of clean reaction process, high efficiency, low energy consumption and less pollutants. The application of microwave technology opens up new ways of drug synthesis. With the continuous development of microwave technology, microwave technology will promote the development of chemical synthesis. Although microwave chemistry still faces many problems to be solved, microwave technology will have great economic value as microwave technology matures.

\section{References}

[1] Zhang Baohua. Microwave technology in the synthesis of materials [J]. Technology Information, 
2015 (11): 17-18.

[2] Yu Guanghui, Zhai Wanzhou. Microwave technology in the synthesis of molecular sieve materials [J]. Silicate Bulletin, 2016, (10): 141-142.

[3] Li Zhibin, Zhang Ling Ke, Fang Yanchun, and so on. Microwave in the synthesis of materials [J]. Industrial Furnaces, 2015, (2): 26-27.

[4] Fan Chengli, Cai Yujing, Xiang Hongmei, et al. Application of Microwave Chemical Reactor in Medicinal Chemistry [J]. Experimental Science and Technology, 2016, (3): 36-37.

[5] Zhang Weizhong, Zhang Bo, Wang Xiufang, and so on. Synthesis and characterization of 2-phenylbenzimidazole under microwave irradiation [J]. Fine Chemicals, 2015, (5): 21-22. 